\title{
$\mathrm{HIGH}-$ \\ LIGHTS
}

URLS

Tom 40

http://db. yeastg

enome.org/cgibin/SGD/locus.pl ? locus $=$ Tom 40

Mas 37

http://db. yeastg

enome.org/cgi-

bin/SGD/locus.pl

? locus $=$ Mas 37

porin

http: //db. yeastg

enome.org/cgi-

bin/SGD/locus.pl

? locus=POR1

$\mathrm{Mdm} 10$

http: //db. yeastg

enome.org/cgi-

bin/SGD/locus.pl

? Iocus $=$ Mdm10

Ugo 1

http: //db. yeastg

enome.org/cgi-

bin/SGD/locus.pl

? locus=Ugo 1

PROTEIN SORTING

\section{Over to you, SAM}

Most mitochondrial proteins are made in the cytosol, so to reach their final destination they need to be transported through the TOM (translocase of the outer mitochondrial membrane) and TIM (translocase of the inner mitochondrial membrane) complexes. In addition to protein transport, it has been thought that these translocases also sort proteins to their sub-mitochondrial destination. However, in Nature, Meisinger and colleagues now reveal that another complex - SAM functions after TOM to sort and assemble complex outer-membrane proteins.

The Tom components of the TOM complex are encoded in the nucleus, so they are imported into mitochondria through TOM. The biogenesis of Tom 40 - which forms the TOM channel - requires nearly all of the TOM subunits, and Meisinger and co-workers began their work by showing that the outer mitochondrial membrane protein Mas37 is also involved in Tom40 biogenesis. Previously, Mas37 has been proposed to be an import receptor and a Tom protein, but it was subsequently found to be neither.

To clarify the role of Mas37, Meisinger and colleagues studied the import of a ${ }^{35} \mathrm{~S}$-labelled precursor of Tom40 into isolated wild-type and mas374 yeast mitochondria. They found that Tom 40 assembly into the core TOM complex occurs through

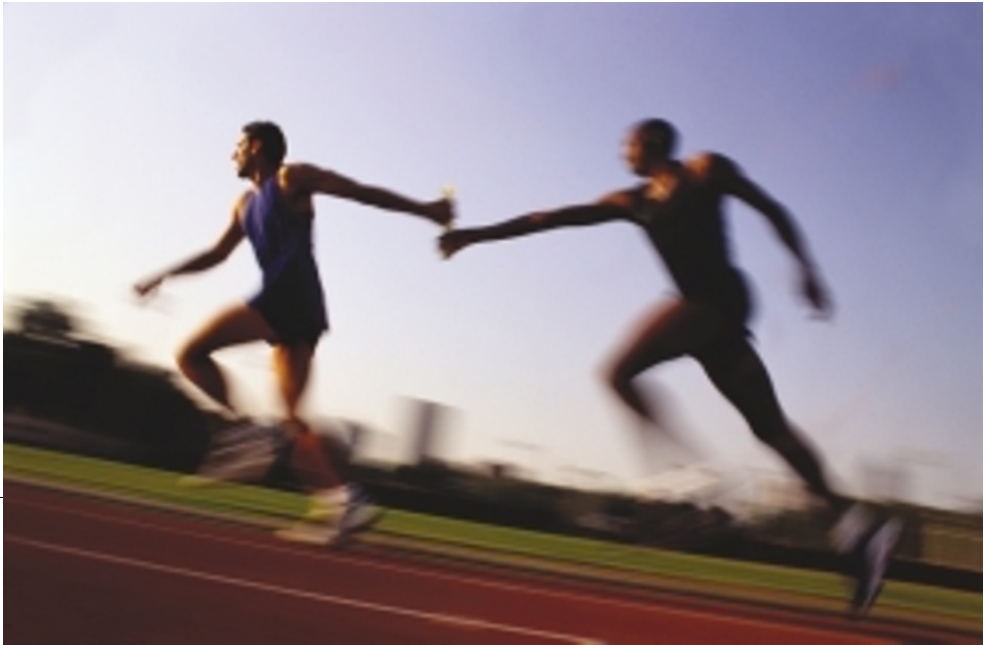

Tom40 assembly intermediates I and II in wild-type mitochondria and that, in mas37 3 mitochondria, the formation of all three complexes was strongly inhibited. The latter effect was also observed after incubating wild-type mitochondria with antiMas37 antibodies. They further showed that the level of the outer mitochondrial membrane protein porin was reduced in mas $37 \Delta$ mitochondria, whereas protein markers of the mitochondrial matrix or inner membrane were unaffected. Mas37 is therefore needed for Tom 40 biogenesis, but not for protein import to the inner membrane.

Next, Meisinger and co-workers studied the import and assembly of three other outer mitochondrial membrane proteins. They found that the assembly of the porin and Mdm10 - integral proteins with predicted $\beta$-strands - was strongly inhibited in mas $37 \Delta$ mitochondria whereas the assembly of Ugol, which has a single transmembrane segment, occurred normally. This indicates that Mas37 is required for the biogenesis of complex, $\beta$-barrel outer-membrane proteins, but not for the biogenesis of relatively simple proteins.

Mas37 forms part of a complex that is devoid of Tom proteins and the authors called this complex SAM, because of its role in the sorting and assembly of outer mitochondrial membrane proteins. They found that mitochondria in which Mas37 has been disrupted accumulate nonassembled Tom 40 precursors.
However, by contrast, they showed that disrupting the Tom proteins that target the Tom 40 precursor does not result in the accumulation of these precursors. This shows that the TOM machinery is needed for initial import before SAM can function.

In the final part of their study, Meisinger and colleagues showed that blocking the general import pore of endogenous TOM greatly impaired the formation of the Tom 40 assembly intermediate I, which, again, indicates that TOM functions before SAM. This work has therefore changed our view of organellar protein sorting. It now seems that the TOM complex, which was previously thought to sort and assemble proteins, “...is not sufficient for the correct integration of outer membrane proteins with a complicated topology, and instead transfers precursor proteins to the SAM complex".

Rachel Smallridge ORIGINAL RESEARCH PAPER Wiedemann, N. et al. Machinery for protein sorting and assembly in the mitochondrial outer membrane. Nature $\mathbf{4 2 4}$, 565-571 (2003)

FURTHER READING Neupert, W. \& Brunner, M. The protein import motor of mitochondria Nature Rev. Mol. Cell Biol. 3, 555-565 (2002) WEB SITE

Nikolaus Pfanner's laboratory: http://www.biochemie.unifreiburg.de/pfanner/pfanner.htm (0) References and links 